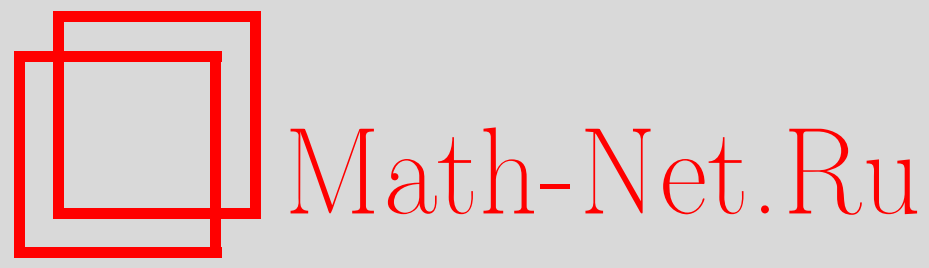

Ю. Аппель, Е. М. Семёнов, Константа упаковки перестановочно-инвариантных пространств, Функи. анализ и его прил., 1998, том 32, выпуск 4, 69-72

DOI: https://doi.org/10.4213/faa429

Использование Общероссийского математического портала MathNet.Ru подразумевает, что вы прочитали и согласны с пользовательским соглашением

http://www . mathnet.ru/rus/agreement

Параметры загрузки:

IP : 3.85 .5 .30

26 апреля 2023 г., 17:05:56

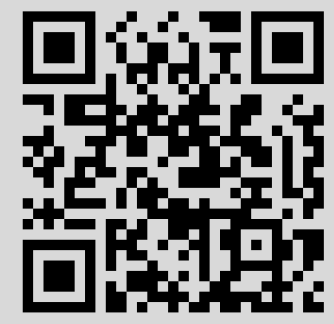




\title{
Константа упаковки перестановочно-инвариантных пространств
}

\author{
(C) 1998. Ю. АпПЕль, Е. М. Семенов
}

Пусть $E$ - бесконечномерное банахово пространство и $B(x, r)$ - замкнутый шар радиуса $r$ с центром в точке $x$. Константой упаковки пространства $E$ называют число

$$
\begin{aligned}
\Lambda(E)=\sup \left\{r: r>0, \exists x_{i} \in E\right. & , i=1,2, \ldots, \\
& \left.\inf _{i \neq j}\left\|x_{i}-x_{j}\right\| \geqslant 2 r, \bigcup_{i=1}^{\infty} B\left(x_{i}, r\right) \subset B(0,1)\right\} .
\end{aligned}
$$

Финитную константу упаковки $\Lambda_{f}(E)$ можно определить аналогичным образом:

$$
\begin{aligned}
\Lambda_{f}(E)=\sup \{r: r>0, \forall n \exists & x_{1}, \ldots, x_{n} \in E, \\
& \left.\min _{i \neq j}\left\|x_{i}-x_{j}\right\| \geqslant 2 r, \bigcup_{i=1}^{n} B\left(x_{i}, r\right) \subset B(0,1)\right\} .
\end{aligned}
$$

Очевидно, что $\Lambda(E) \leqslant \Lambda_{f}(E)$. В [1] было показано, что

$$
\Lambda(E)=D(E)(2+D(E))^{-1} \text {, }
$$

где

$$
D(E)=\sup _{\left\|x_{i}\right\| \leqslant 1} \inf _{1 \leqslant i \neq j<\infty}\left\|x_{i}-x_{j}\right\|_{E} .
$$

Финитным аналогом этой характеристики является константа

$$
D_{f}(E)=\lim _{n \rightarrow \infty} \sup _{\left\|x_{i}\right\| \leqslant 1,1 \leqslant i \leqslant n} \min _{1 \leqslant i \neq j \leqslant n}\left\|x_{i}-x_{j}\right\| .
$$

Очевидно, что $D(E) \leqslant D_{f}(E)$.

Константа $\Lambda\left(l_{2}\right)$ была найдена в [2]. Этот результат был распространен в [3] на пространства $l_{p}: \Lambda\left(l_{p}\right)=\left(1+2^{1-1 / p}\right)^{-1}$ для $1 \leqslant p<\infty$. Если мера пространства неатомична и $1 \leqslant p \leqslant 2$, то $\Lambda\left(L_{p}\right)=\left(1+2^{1-1 / p}\right)^{-1} ;$ если $2 \leqslant p<\infty$, то $\Lambda\left(L_{p}\right)=\left(1+2^{1 / p}\right)^{-1}$ [4]. Изучению связи между константой упаковки и рефлексивностью пространства посвящены работы $[1,5]$. Нижние и верхние оценки константы упаковки в пространствах Орлича были получены в работах [6-9]. Минимальный радиус шара, содержащего такие $x_{1}, \ldots, x_{n} \in l_{p}$, что $\left\|x_{i}-x_{j}\right\| \geqslant 1$ для всех $1 \leqslant i \neq j \leqslant n$, был найден

* Работа второго автора была частично поддержана РФФИ (грант 95-01-00135) и DAAD (грант A/97/52487). Он искренне признателен Математическому институту университета Вюрцбурга за гостеприимство, оказанное ему в июне-июле 1997 г. 
в [10]. Для любого бесконечномерного пространства $E$ справедливо неравенство $D(E)>1$ [11]. Поэтому $1 / 3<\Lambda(E) \leqslant 1 / 2$. Настоящая работа посвящена изучению константы упаковки перестановочно-инвариантных пространств.

Банахово пространство $E$ измеримых на $[0,1]$ функций называется перестановочно-инвариантным (r. і.) или симметричным, если $x^{*}(t) \leqslant y^{*}(t)$ и $y \in$ $E$ влечет за собой $x \in E$ и $\|x\|_{E} \leqslant\|y\|_{E}$, когда $x^{*}(t)$ не возрастает на $(0,1]$ и равноизмерима с $|x(t)|$. Следуя [11], мы будем предполагать, что $E$ сепарабельно или изометрично сопряженному пространству. Пространства Лоренца $L_{p, q}$ с квазинормой

$$
\|x\|_{L_{p, q}}= \begin{cases}\left(\frac{p}{q} \int_{0}^{1}\left(x^{*}(t) t^{1 / p}\right)^{q} \frac{d t}{t}\right)^{1 / q}, & 1 \leqslant q<\infty, \\ \sup _{0<t \leqslant 1} x^{*}(t) t^{1 / p}, & q=\infty,\end{cases}
$$

доставляют содержательный пример r.i. пространств; здесь $1<p<\infty$, $1 \leqslant q \leqslant \infty$. Квазинорма (2) есть норма при $1 \leqslant q \leqslant p$ и эквивалентна норме при $q>p$. Теория r. i. пространств изложена в монографиях $[12,13]$. Формула (1) позволяет свести изучение константы упаковки к изучению более наглядной константы $D(E)$. Поэтому в дальнейшем мы будем интересоваться лишь свойствами констант $D(E)$ и $D_{f}(E)$.

Пусть $\left(E_{0}, E_{1}\right)$ 一 пара r.i. пространств и $0 \leqslant \theta \leqslant 1$. Через $\left[E_{0}, E_{1}\right]_{\theta}$ обозначаются пространства Кальдерона, построенные по этой паре, с нормой

$$
\|x\|_{\theta}=\inf _{\left\|x_{0}\right\|_{E_{0}}=\left\|x_{1}\right\|_{E_{1}}=1} \sup _{t} \frac{|x(t)|}{\left|x_{0}(t)\right|^{1-\theta}\left|x_{1}(t)\right|^{\theta}} .
$$

Следуя [14], r. i. пространство $E$ назовем $\theta$-гильбертовым, если существует такое r.i. пространство $E_{0}$, что $E=\left[E_{0}, L_{2}\right]_{\theta}$. Это равенство означает совпадение пространств как множеств и равенство норм.

Теорема 1. Пусть $0<\theta<1$. Если r.i. пространство Е является $\theta$-гильбертовьлм, то

$$
D_{f}(E) \leqslant 2^{1-\theta / 2} \text {. }
$$

Хорошо известно, что пространство $L_{p}$ является $\theta$-гильбертовым, где $\theta=$ $2 \min (1 / p, 1-1 / p)$. Так как

$$
D\left(L_{p}\right)=2^{\max (1 / p, 1-1 / p)}
$$

[4], то это соотношение означает, что (3) для пространств $L_{p}$ обрашается в равенство. Подобная конструкция была использована в [15] для оценки константы Юнга.

При эквивалентных перенормировках $E$ константа $D(E)$ может меняться. Например, если $E$ есть $L_{2}$ с нормой

$$
\|x\|_{E}=\left(\int_{0}^{1 / 2}\left(x^{*}(t)\right)^{2} d t\right)^{1 / 2}
$$


то нормы в $E$ и $L_{2}$ эквивалентны. Однако $D(E)=2$. В связи с этим рассмотрим характеристики

$$
G(E)=\inf D(F), \quad G_{f}(E)=\inf D_{f}(F),
$$

где inf берется по всем эквивалентным перестановочно-инвариантным перенормировкам $F$ пространства $E$. Напомним определения $p$-выпуклости и $q$-вогнутости банаховой решетки [12]. Пусть $1 \leqslant p, q \leqslant \infty$. Банахова решетка $E$ называется $p$-выпуклой ( $q$-вогнутой), если для некоторого $C>0$, любого натурального $n$ и любых $x_{1}, \ldots, x_{n} \in E$ выполнено неравенство

$$
\begin{gathered}
\left\|\left(\sum_{i=1}^{n}\left|x_{i}\right|^{p}\right)^{1 / p}\right\| \leqslant C\left(\sum_{i=1}^{n}\left\|x_{i}\right\|^{p}\right)^{1 / p} \\
\left(\left\|\left(\sum_{i=1}^{n}\left|x_{i}\right|^{q}\right)^{1 / q}\right\| \geqslant C\left(\sum_{i=1}^{n}\left\|x_{i}\right\|^{q}\right)^{1 / q}\right) .
\end{gathered}
$$

ТЕОРема 2. Пусть r. $і$. пространство Е является р-вьпукльим и q-вогнутылм для некоторьх $1<p \leqslant q<\infty$. Тогда

$$
G_{f}(E) \leqslant 2^{\max (1 / p, 1-1 / q)} .
$$

Отсюда вытекает, что

$$
G_{f}(E) \leqslant 2^{\max (1 / p(E), 1-1 / q(E))},
$$

где $p(E)$ есть точная верхняя грань тех $p \geqslant 1$, для которых $E p$-выпукло, и $q(E)$ равно точной нижней грани тех $q \leqslant \infty$, для которых $E q$-вогнуто.

Рассмотрим в качестве примера пространства $L_{p, q}$. Известно [16], что $L_{p, q} \min (p, q)$-выпукло и $\max (p, q)$-вогнуто. В силу теоремы 2

$$
G_{f}\left(L_{p, q}\right) \leqslant 2^{\max (1 / p, 1-1 / p, 1 / q, 1-1 / q)} .
$$

Нижние оценки для $D(E)$ получаются в других теоремах. Положим

$$
\gamma(E)=\sup \left\{\gamma: \gamma \geqslant 0, t^{-\gamma} \in E\right\} .
$$

Очевидно, что $0 \leqslant \gamma(E) \leqslant 1$. Например, $\gamma\left(L_{p, q}\right)=1 / p$ для всех $1<p<\infty$, $1 \leqslant q \leqslant \infty$.

ТЕОРемА 3. Если $E-$ r. i. пространство, то

$$
D(E) \geqslant 2^{\max (\gamma(E), 1-\gamma(E))} \text {. }
$$

Теоремы 2,3 и результаты [17] позволяют вычислить $G_{f}\left(L_{p, q}\right)$ и оценить $G\left(L_{p, q}\right)$. Справедлива

Teорема 4. Пусть $1<p<\infty, 1 \leqslant q \leqslant \infty$. Тогда

$$
2^{\max (1 / p, 1-1 / p, 1 / q)} \leqslant G\left(L_{p, q}\right) \leqslant G_{f}\left(L_{p, q}\right)=2^{\max (1 / p, 1-1 / p, 1 / q, 1-1 / q)} .
$$

Мы предполагаем, что $G\left(L_{p, q}\right)$ совпадает с левой частью формулы $(5)$. Однако нам не удалось доказать эту гипотезу. Заметим, что $G\left(l_{q}\right)<G_{f}\left(l_{q}\right)$ для $2<q<\infty$. 


\title{
ЛИТЕРАТУРА
}

1. Kottman C. A. Trans. Am. Math. Soc., 150, 565-576 (1970). 2. Rankin R. A. Proc. M. A. Glasgow, 2, 139-144 (1955). 3. Burlak J. A. C., Rankin R. A., Robertson A. P. Proc. M. A. Glasgow, 4, 22-25 (1958). 4. Wells J. H., Williams L. R. Imbeddings and extensions in analysis, Springer-Verlag, Berlin, 1975. 5. Hudzik $H$. Collect. Math., 44, 131-135 (1993). 6. Clever C. E. Pacific J. Math., 65, 325-335 (1976). 7. Rao M. M., Ren Z. D. Theory of Orlicz spaces, M. Dekker, N.Y., 1991. 8. Hudzik H., Landes T. Bull. U.M.I., (7) 9-A, 225-237 (1995). 9. Rao M. M., Ren Z. D. Studia Math., 126, No. 3, 235-251 (1997). 10. Ball K. Israel J. Math., 59, 243-256 (1987). 11. Elton J., Odell E. Colloq. Math, 44, 105-109 (1981). 12. Lindenstrauss J., Tzafriri L. Classical Banach spaces. Function spaces. II, Springer-Verlag, Berlin, 1979. 13. Крейн С. Г., Петунин Ю. И., Семенов Е. М. Интерполяция линейных операторов, Наука, M., 1978. 14. Pisier G. J. d'Analyse Math., 35, 264-281 (1979). 15. Franchetti C., Semenov E. M. C. R. Acad. Sci. Paris, 323, 723-728 (1996). 16. Carothers N. L., Dilworth S. J. Texas Func. Anal. Sem., 1985-1986, pp. 107-133. 17. Figiel T., Johnson W. B., Tzafriri L. J. Approx. Theory, 13, 395-412 (1975).

Университет Вюрцбурга

Воронежский государственный университет
Поступило в редакцию 10 февраля 1998 г.

УДК 517.9

\section{Собственные значения оператора теории оболочек в зоне регулярного вырождения}

\author{
(C) 1998. А. Г. АСЛАНЯН
}

Рассматриваются свободные колебания тонкой упругой оболочки произвольного очертания. Срединная поверхность оболочки $S$ предполагается выпуклой и достаточно гладкой, ее граница $\gamma$ - кусочно гладкой. На поверхности $S$ вводится ортогональная система координат $\alpha, \beta$, отнесенная к линиям кривизны $((\alpha, \beta) \in S)$. Указанные колебания описываются системой трех дифференциальных уравнений в перемещениях [1]

$$
\left(\mu^{4} N+L\right) u=\lambda u \text {. }
$$

Здесь $N$ и $L$ - матричные дифференциальные операторы; их явный вид ввиду громоздкости мы не приводим (см., например, [1, с. 8; 40, 41]). Заметим, что вид оператора $N$ зависит от выбора соотношений упругости в теории оболочек [2, с. 11]. Однако сформулированный ниже результат справедлив во всех наиболее часто встречающихся вариантах соотношений упругости (см. $[3$, c. $299 ; 4$, c. $55-58])$.

Далее, $u(\alpha, \beta)=\left(u_{1}, u_{2}, u_{3}\right)$ - вектор перемещения точки срединной поверхности оболочки (компонента $u_{3}$ направлена по нормали к поверхности $S$, а компоненты $u_{1}, u_{2}$ лежат в касательной плоскости), $\lambda$ - спектральный параметр, пропорциональный квадрату собственной частоты $\omega$, и $\mu$ - малый параметр, $\mu^{4}=h^{2} / 12$, где $h$ - толщина оболочки. 